\title{
Titanium dioxide-reduced graphene oxide thin film for photoelectrochemical water splitting
}

\begin{abstract}
The incorporation of reduced graphene oxide ( $\mathrm{rGO}$ ) on a $\mathrm{TiO} 2$ surface had been demonstrated to be an effective method to enhance the photoelectrochemical performance. A $\mathrm{TiO} 2 \mathrm{II}$ rGO thin film was fabricated by depositing TiO2 on ITO using an aerosol-assisted chemical vapor deposition method and GO dip-coating, followed by thermal reduction of the GO layer. The fabricated thin film was characterized using XRD and FESEM techniques. The photoelectrotrochemical performance of the TiO2ï rGO thin film was investigated under the illumination of simulated solar light. The TiO2ï rGO showed a higher photocurrent response $(80.2 \mu \mathrm{A})$ than bare $\mathrm{TiO} 2(13.1 \mu \mathrm{A})$. This improved photoelectrochemical performance was due to the $\mathrm{rGO}$, which increased the electron transport and thereby minimized the charge recombination process. The TiO2ï rGO thin film showed good stability, even after being subjected to 1000 voltammetric cycles, and the rGO sheets remained adhered to the surface of the $\mathrm{TiO} 2$ thin film.
\end{abstract}

Keyword: Graphene; Photoelectrochemistry; Titanium oxide; Thin film; Water splitting 